\title{
Actitud del docente hacia la enseñanza de competencias de investigación
}

\section{Attitude of the teachers towards the teaching of Research Competences}

\author{
Jean Jiménez \\ jean_jimenez22@hotmail.com
}

Universidad Nacional Experimental de los Llanos Occidentales “Ezequiel Zamora”, Barinas, Venezuela

\section{RESUMEN}

El propósito de esta investigación fue determinar la actitud del docente hacia la enseñanza de competencias investígativas a los estudiantes de las instituciones educativas, ubicadas en el municipio Obispos, estado Barinas. La investigación se desarrolla mediante el método holopráxico de la comprensión holística de la ciencia. El tipo de investigación es descriptiva con un diseño de campo, transeccional contemporáneo unieventual. La unidad de estudio estuvo conformada por 70 profesores de 3 instituciones educativas del municipio Obispos, estado Barinas. Con respecto al abordaje es cosmológico, exógeno y etic. Para la recolección de los datos se utiliza la técnica de la encuesta con un instrumento cuestionario el cual se confíabiliza mediante la técnica esta-dística Alfa de cronbach, la cual arroja un valor de 0,78 . Para el análisis de los datos se utiliza la estadística descriptiva. Las conclusiones descritas denotan que los docentes consultados se manifiestan verbal, emocional y conductualmente a favor de la enseñanza de competencias en investigación.

Palabras clave: Holística; perceptivo; educación; competencias, investigación

\begin{abstract}
The purpose of this research is to determine the attitude of the teacher toward teaching investigative skills to students of educational institutions, located in the municipality of Obispos, Barinas State. The research is developed through the holopraxic method of holistic understanding of science. The type of research is descriptive with a field design, transactional contemporary unieventual. The study unit is made up of 70 teachers from three educational institutions in the municipality of Obispos, Barinas State. Regarding the approach, it is cosmological, exogenous and etic. To collect the data, the survey technique was used with a questionnaire instrument, which was made reliable using the Alfa de Cronbach statistical technique, which yields a value of 0.78. For the analysis of the data, descriptive statistics are used. The conclusions described show that teachers manifested verbally, emotionally and behaviorally in favor of teaching research skills.
\end{abstract}

Key words: Holistic; perceptive; education; competencies, research 


\section{INTRODUCCIÓN}

La educación es considerada uno de los pilares fundamentales para la transformación integral de los individuos, debido a que a través de ella se ofrecen un conjunto de posibilidades pedagógicas que permiten el desarrollo potencial de los aspectos referidos a lo afectivo, intelectual, cognitivo, y social que sirven como herramienta de apoyo para la toma de decisiones efectivas y participar de forma plena en todas las actividades no solo de carácter escolar sino también en el contexto cotidiano.

Dentro de sus planteamientos filosóficos aparece la enseñanza de las competencias en investigación como una acción didáctica destinada a potenciar el saber hacer, y saber actuar con base en un conocimiento, donde el ser humano se convierte en un agente activo para generar soluciones a un determinado contexto.

La enseñanza de competencias investigativas en el ámbito escolar implica que el estudiante aprenda integralmente como a través de sus cualidades puede observar, preguntar, argumentar y sistematizar adecuadamente lo vivencial, y explicar el mundo social en el que vive; esto le interconecta con aspectos esenciales de su vida, cognitivo: comprensión e interiorización de saberes, conductual: práctica de habilidades/destrezas y afectivo: el ser como atributo importante de participación activa en las situaciones contextuales.

Para ello, el docente necesita revisar su cosmovisión como gestor de aprendizaje para generar una actitud que le permita transcender de lo eminentemente académicotradicional hacia un planteamiento educativo holístico que reconozca las potencialidades del estudiante como un ser integral capaz de desarrollar capacidades que afiancen el espíritu investigativo, así como la construcción de bases sólidas para la vida al poner en práctica bajo una perspectiva crítica los saberes aprendidos en su cotidianidad, en aras de proponer, mediante el trabajo científico, soluciones a las problemáticas sociales.

En ocasiones esto no se da y la enseñanza de competencias investigativas se ve afectada por la actitud no favorable hacia el desarrollo de la misma en el aula de clase, lo cual no produce los resultados que espera una sociedad del conocimiento ávida de hombres y mujeres educados para la innovación y la creatividad, de allí que se necesite una enseñanza que tenga en cuenta las competencias investigativas, donde el estudiante aprenda a involucrarse con autonomía a una realidad concreta, donde sus competencias saber ser, saber hacer, saber conocer y saber convivir, le permitan abordar desde lo ético y lo creativo, problemáticas de desarrollo social $\mathrm{y}$ aplicar su conocimiento inter y transdisciplinar en su manejo con un pensamiento lógico, crítico y reflexivo; y responder así de manera asertiva a las necesidades de su entorno (Tobón, 2008, p. 41).

Con base a lo descrito, el presente artículo tiene como objetivo central dar respuesta a la actitud del docente hacia la enseñanza de competencias investigativas en los estudiantes de las instituciones educativas, ubicadas en el municipio Obispos, estado Barinas.

\section{MÉTODO}

Este estudio se considera de tipo descriptivo por cuanto en ella se buscó describir las características, atributos, condiciones de los docentes objeto de estudio, es así como Álvarez, (2005), señala que el tipo de investigación descriptiva es aquella que tiene como finalidad o propósito la descripción o caracterización de un objeto dado en su entorno natural. (p. 51). 
De acuerdo con el criterio de amplitud de la información a recolectar, es de campo, por cuanto los datos de interés se recogen a partir de fuentes vivas, es decir, de los docentes que dictan sus cátedras en el nivel de media general, los cuales se encuentran inmersos en el ambiente natural donde ocurre el evento de estudio. En lo que respecta a la perspectiva temporal, es contemporáneo transaccional, debido que se estudia el evento en un periodo de tiempo dado para encontrar las características propias que lo definen. Hernández y otros (2010), señalan que el diseño transeccional se basa en recopilar los datos en un solo momento, en un tiempo único.

En cuanto a la amplitud y organización de los datos, el evento es de carácter univariable o unieventual, porque "la recolección de los datos está focalizada en un único evento, ya sea para describirlo, compararlo, analizarlo, explicarlos"... (Hurtado, 2012, p. 695).

De conformidad con Hurtado (2012), se estudia como único evento la actitud del docente hacia la enseñanza de competencias en investigación para conocer la manifestación en sus tres dimensiones: cognitiva, afectiva y conductual frente al objeto mencionado.

La población de estudio estuvo conformada por setenta profesionales de la docencia que participan del proceso educativo, es decir, aquellas personas encargadas de la enseñanza aprendizaje de los contenidos curriculares.

En la recolección de los datos se utiliza la técnica de la encuesta mediante un instrumento de recolección de información denominado: escala de actitudes hacia la enseñanza de competencias investigativas, con cinco (5) ítems o formas de respuestas vinculadas a las sinergias tales como: cognitiva, afectiva y conductual, (TA), Totalmente de acuerdo (PA), Parcialmente de acuerdo, (I), Indiferencia, (PD), Parcialmente en desacuerdo, (TD), Totalmente en desacuerdo.

La confiabilidad del instrumento se calculó con alfa de cronbach mediante el programa estadístico SPSS 18 lo cual da un resultado de 0,78 , lo cual indica que el instrumento fue confiable. En el análisis de los datos se utiliza la estadística descriptiva específicamente la mediana porque los datos se miden en un nivel ordinal.

\section{RESULTADOS Y DISCUSIÓN}

Los datos arrojados relacionados en el desarrollo de este estudio fueron producto del procesamiento de la información obtenida al aplicar el instrumento de investigación a setenta docentes que imparten las cátedras o asignaturas en el nivel del subsistema de secundaria de las instituciones: Unidad Educativa Nacional Manuel Palacios, Liceo Bolivariano Borburata y Escuela Técnica Manuel Palacios, ubicadas en el municipio Obispos, del estado Barinas.

Para una mejor comprensión se establece un baremo de interpretación, en el cual se especifica el rango del valor desarrollado en una escala de intervalo que va de 0 a 50 puntos según los puntajes transformados, así como también las categorías que corresponden a esos rangos. Posteriormente se presenta el análisis global del evento actitud docente hacia la enseñanza de competencias investigativas en la muestra estudiada. (Tabla 1). 
Tabla 1. Baremo de interpretación de puntajes

\begin{tabular}{cc}
\hline Rango & Categoría \\
\hline $0-9,99$ & Muy negativa \\
$10-19,99$ & Negativa \\
$20-29,99$ & Indiferente \\
$30-39,99$ & Positiva \\
$40-50$ & Muy positiva \\
\hline
\end{tabular}

Los resultados del análisis global del evento actitud hacia la enseñanza de las competencias investigativas se presentan en la tabla 2 donde se observa que la mediana obtenida de 32,2 puntos sobre un máximo de 50 puntos, se ubica específicamente en la categoría actitud positiva según el baremo de interpretación, es decir, que la actitud del docente hacia la enseñanza de competencias investigativas es positiva, lo cual implica que los docentes en su proceso educativo muestran una tendencia favorable hacia el acercamiento del objeto de estudio que generalmente está unido a la opinión, aceptación y disposición frente a lo que enseña.

Tabla 2. Estadísticos de Actitud hacia la enseñanza de la investigación

\begin{tabular}{lcl}
\hline $\mathbf{N}$ & Válidos & $\mathbf{7 0}$ \\
\hline Mediana & & 32,2 \\
Mínimo & & 27,2 \\
Máximo & & 41,3 \\
Percentiles & 25 & 30,8 \\
& 50 & 32,2 \\
& 75 & 35,7 \\
\hline
\end{tabular}

El gráfico 1, refleja un puntaje mínimo de 27,27 y un máximo de 41,36. La distribución es bastante asimétrica, sin embargo el grupo que está debajo de la mediana presenta características más comunes que el grupo que está por encima. A continuación, se describe la distribución de los docentes en cada categoría de actitud hacia la enseñanza de competencias en investigación. 


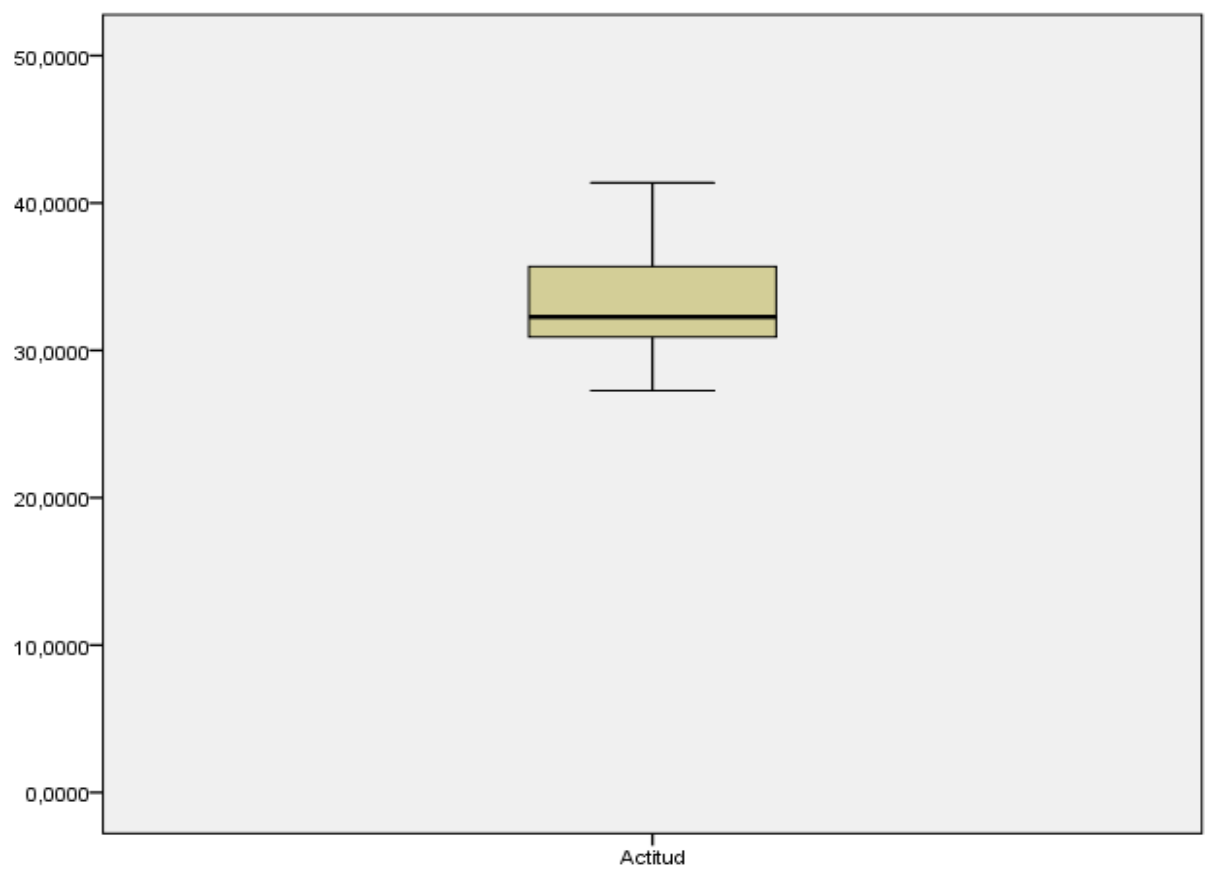

Gráfico 1. Caja y bigote del evento actitud docente hacia la enseñanza de competencias investigativas

Para representar como se distribuye la población en las categorías del evento actitud hacia la enseñanza de competencias investigativas, se presentan en la tabla 3, donde se observa que el mayor porcentaje se concentra en la categoría positiva, con un $81,4 \%$, en segundo lugar, aparece la categoría indiferente con un $17,1 \%$, mientras que la categoría muy positiva se ubica en un valor de $1,4 \%$, lo que permite destacar una tendencia con respecto a la actitud del docente hacia la enseñanza de competencias investigativas que va entre la categoría indiferente a positiva con una sumatoria de ambas de $88,5 \%$.

Tabla 3. Categoría de actitud hacia la enseñanza de las competencias en investigación

\begin{tabular}{llcccc}
\hline & Frecuencia & Porcentaje & $\begin{array}{c}\text { Porcentaje } \\
\text { válido }\end{array}$ & $\begin{array}{c}\text { Porcentaje } \\
\text { acumulado }\end{array}$ \\
\hline Validos & Indiferente & 12 & 17,1 & 17,1 & 17,1 \\
& Positiva & 57 & 81,4 & 81,4 & 98,6 \\
& Muy positiva & 1 & 1,4 & 1,4 & \\
& Total & 70 & 100,0 & 100,0 & 100,0 \\
\hline
\end{tabular}

Los resultados inducen a afirmar que los docentes pertenecientes a las instituciones educativas señaladas en el estudio tienen una actitud positiva referido a la enseñanza de competencias en investigación, donde incluye aspectos intelectivos, observacionales, meto- dológicos, analíticos y escriturales dentro de su ejercicio educativo. Además, que el evento está conformado por tres sinergias como lo son la opinión, aceptación y disposición. (Tabla 4). 
Tabla 4. Sinergias del evento

\begin{tabular}{lccc}
\hline & Opinión & Emoción & Disposición \\
\hline N Válidos & 70 & 70 & 70 \\
& & & \\
Mediana & 33,07 & 31,59 & 32,69 \\
Mínimo & 26,56 & 24,30 & 26,92 \\
Máximo & 42,18 & 43,75 & 39,42 \\
Percentiles 25 & 31,25 & 28,47 & 30,76 \\
50 & 33,07 & 31,59 & 32,69 \\
75 & 35,93 & 34,89 & 35,57
\end{tabular}

\section{Análisis de las sinergias presentes en el evento actitud del docente hacia la enseñanza de competencias inves- tigativas}

Con relación a los resultados de las sinergias opinión, emoción y conductual del evento actitud hacia la enseñanza de competencias investigativas, (cuadro 4), se aprecia para la sinergia de la dimensión cognitiva una mediana de 33,0 puntos de un máximo de 50 puntos, lo cual hace que se ubique dentro del baremo en la categoría de positiva, ante lo que se puede afirmar que los docentes tienen una buena opinión sobre la enseñanza de competencias investigativas. La distribución del puntaje en esta sinergia es bastante simétrica aunque el grupo que está por debajo de la mediana es más homogéneo que el que se encuentra por encima. No se observan casos atípicos.

Además, la distribución de los casos de la sinergia componente cognitivo u opinión que se presenta en la tabla 5, indica que un $80 \%$ de los docentes manifiestan una actitud positiva, lo que significa que existe un valor considerable de docentes que poseen una actitud favorable. Mientras que un 15,7\%, se encuentra en la categoría indiferente, otro valor importante está representado por un $4,3 \%$, de los docentes que poseen una actitud muy positiva.

Tabla 5. Categoría del componente cognitivo

\begin{tabular}{llcccc}
\hline & Frecuencia & Porcentaje & $\begin{array}{c}\text { Porcentaje } \\
\text { válido }\end{array}$ & $\begin{array}{c}\text { Porcentaje } \\
\text { acumulado }\end{array}$ \\
\hline Válidos & Indiferente & 11 & 15,7 & 15,7 & 15,7 \\
& Positiva & 56 & 80,0 & 80,0 & 95,7 \\
& Muy positiva & 3 & 4,3 & 4,3 & 100,0 \\
& Total & 70 & 100,0 & 100,0 & \\
\hline
\end{tabular}

Con respecto a la sinergia componente emoción, donde se obtuvo una mediana de 31,5 puntos (ver tabla 4), la cual se ubica según el baremo de interpretación en una categoría positiva indica que los docentes tienen una aceptación favorable sobre la enseñanza de competencias en investigación. La distribución de los datos se presenta de forma simétrica, los puntajes del grupo que está por encima de la mediana son más dispersos que los que están por debajo. No se observan casos atípicos
La distribución de los casos de la sinergia componente emoción o aceptación se presentan en tabla 6 , en la cual se describe que un $68,6 \%$ de los docentes manifiestan una actitud de aceptación positiva, esto indica que los docentes tiende a concentrar una aceptación favorable con respecto a la enseñanza de competencias investigativas. Un $30 \%$, manifestó sentirse indiferente, por último, el 1,4\% expreso se ubicó en la categoría muy positiva. 
Tabla 6. Categoría componente emoción

\begin{tabular}{llcccc}
\hline & Frecuencia & Porcentaje & $\begin{array}{c}\text { Porcentaje } \\
\text { válido }\end{array}$ & $\begin{array}{c}\text { Porcentaje } \\
\text { acumulado }\end{array}$ \\
\hline Válidos & Indiferente & 21 & 30,0 & 30,0 & 30,0 \\
& Positiva & 48 & 68,6 & 68,6 & 98,6 \\
& Muy positiva & 1 & 1,4 & 1,4 & 100,0 \\
& Total & 70 & 100,0 & 100,0 & \\
\hline
\end{tabular}

Los resultados de la sinergia disposición, se muestra una mediana de 32,69 puntos (ver tabla 4), la cual se ubica según el baremo de interpretación en una categoría positiva, lo cual indica que los docentes tienen una disposición favorable que está en correspondencia con la enseñanza de competencias en investigación. La distri-bución se presenta de forma simétrica, los puntajes del grupo están por encima de la mediana son más dispersos que los que están por debajo. No se observan casos atípicos.

En atención a la distribución de la población en las categorías de la sinergia disposición se observa en la tabla 7 que un $90 \%$ de los docentes consultados poseen una actitud positiva, por lo que se puede resumir que es un indicador favorable en la enseñanza de competencias investigativas. Adicionalmente, un grupo restante de $10 \%$ expresan una actitud indiferente a dicha actividad.

Tabla 7. Categoría componente disposición

\begin{tabular}{cccccc}
\hline & & Frecuencia & Porcentaje & $\begin{array}{c}\text { Porcentaje } \\
\text { válido }\end{array}$ & $\begin{array}{c}\text { Porcentaje } \\
\text { acumulado }\end{array}$ \\
\hline \multirow{3}{*}{ Válidos } & Indiferente & 7 & 10,0 & 10,0 & 10,0 \\
& Positiva & 63 & 90,0 & 90,0 & 100,0 \\
& Total & 70 & 100,0 & 100,0 & \\
\hline
\end{tabular}

\section{Discusión}

\section{Fundamentos teóricos}

La educación como principal medio de socialización propicia la formación integral de las capacidades volitivas, afectivas $y$ biopsicosociales del ser humano; al mismo tiempo abre la posibilidad de establecer acciones pedagógicas orientadas a fortalecer las competencias de aprendizaje para el saber hacer, el saber conocer, el saber ser, y el saber convivir de cada persona como bases fundamentales para el desenvolvimiento pleno dentro de su contexto.

En este sentido, se incluye dentro de las competencias a desarrollar las investigativas, puesto que hacer ciencia o generar conocimiento requiere de individuos con for- mación científica para preguntar, discernir, identificar, problematizar, organizar y construir representaciones que le ayuden a derivar conclusiones efectivas sobre los eventos que surgen de su realidad.

El tema de la enseñanza de competencias investigativas ha sido tratado, por Barreiro (2015), en un estudio sobre las competencias investigativas del estudiante en el proceso de enseñanza aprendizaje en el que revela el poco interés que tienen los profesores por la práctica de la investigación científica en el proceso de enseñanza y aprendizaje. Aunado a ello, señala la inexistencia de estrategias; el desconocimiento de técnicas de búsqueda, identificación, recolección y procesamiento de la información documental lo que permite visualizar una marcada insuficiencia en el 
desempeño investigativo de los estudiantes al momento de desarrollar y presentar tareas de investigación (p. 62).

Otro hallazgo de interés, es el planteado por Acosta (2013), en su investigación acerca de las actitudes investigativas en la formación escolar, resalta las dificultades que presenta la enseñanza de la investigación, la cual, en edad escolar, está influenciada por factores que afectan la investigación formativa y la apropiación de una actitud favorable. Adicionalmente, agrega que la influencia de paradigmas como el positivismo tradicional pedagógico en los procesos de enseñanza, que establece brechas entre la construcción de un conocimiento y la conciencia de una actitud investigativa en los escolares, crea estilos pasivos de aprendizaje investigativos que fragmentan el conocimiento como totalidad.

Al respecto, se conceptúa el término competencia como un proceso complejo constituido por las dimensiones cognitivas, procedimentales y actitudinales (Tobón, 2006), las cuales operan el quehacer funcional del ser humano por las tareas del entorno, a su vez, amplían el sentido crítico y ulterior para la apropiación y aplicación de saberes en un escenario escolar, institucional y social.

Por su parte, según Muñoz y otros (2011), las competencias abarcan una gama de conocimientos, habilidades y actitudes aplicados en el desempeño de una función productiva o académica, dicho de otro modo, refieren el conjunto de saberes integrados al conocer, hacer y al ser que establecen un canal de comunicación entre el interés del individuo $y$ el saber requerido para comprender las complejas relaciones sociales del mundo, de ahí que la escuela se constituye en el espacio más cercano y concreto para enseñar a investigar.

Cabe afirmar que la categoría competencia, tiene dentro de su acepción múltiples definiciones, según su utilidad en el escenario disciplinar, y en el caso propio de la investigación, se hace énfasis a las competencias investigativas como la capacidad de dominio teórico y práctico para preguntar, observar, escribir, analizar, aplicar técnicas e instrumentos diversos en concordancia con la realidad contextualizada del centro educativo, sus necesidades y problemas (Finol y otros, 2008).

Desde otra perspectiva, la idea de competencias investigativas alude al conjunto de capacidades y destrezas que integran elementos del saber-hacer de las personas, los cuales sirven para abordar problemas de contexto, indagar en la revisión de literatura, realizar el análisis de la situaciones problemáticas, y establecer alternativas para su solución (Reyes, 2013). De hecho, las competencias investigativas abren la posibilidad de que se integre un conjunto de procesos para precisar problemáticas del entorno con sentido crítico para dar explicaciones, y argumentar sobre lo que hacen en el escenario social.

Del mismo modo, es el conjunto de disposiciones para la indagación sistemática, con aptitud para precisar los problemas de investigación, y de emplear procedimientos, técnicas, y métodos de acuerdo a la naturaleza de los objetos de investigación, así como habilidades de orden lingüística para argumentar el proceso de lo que se investiga (Palacios y otros, 2013, p. 585).

En concordancia con el autor, las competencias investigativas dan lugar a la formación de habilidades cognitivas, afectivas y accionarias que facilitan la resolución del trabajo científico, al no solo permitir el cumplimiento de las tareas diarias del ser humano, sino que al mismo tiempo habilita nuevas formas de conocer desde una posición sistémica. 
Las competencias investigativas son, entonces, el conjunto de conocimientos que, al ser adquiridos o perfeccionados por el alumno, lo capacitan para cumplir las funciones y tareas específicas en el quehacer investigativo (Pérez, y otros, 2012). Estas competencias responden directamente a la idea de saber utilizar el conocimiento de manera adecuada, definiendo habilidades $\mathrm{y}$ destrezas para observar, argumentar, sistematizar, a fin de crear o gestionar el conocimiento, sobre la base del interés, la motivación hacia la investigación, el desarrollo de capacidades y la realización personal del estudiante (Pérez, 2012).

Entre las diversas tipologías de la enseñanza de competencias, (Muñoz y otros, Finol y otros, 2008), establecen las de orden investigativas que incluyen la pregunta, observación, análisis, así como las metodológicas, las cuales se presentan a continuación:

Competencias para preguntar: inician con el establecimiento de análisis, destacando que las formulaciones constituyen una de las principales herramientas del investigador en su intento de acercarse a la realidad natural del evento. Las competencias para preguntar son un atributo que se construye con el hombre, con su devenir, y en ese sentido cuestiona, indaga, busca respuestas acerca de lo que ocurre en su realidad, para ello dispone de su pensamiento para realizar la cuestión; esta competencia sirve no solo para iniciar el proceso de conocer el evento, sino para discernir las pautas que conducen a cristalizar su desarrollo (Muñoz y otros, 2011).

Competencias observacionales: la observación constituye el principal procedimiento de orden empírico que se aplica a cualquier situación contextual. Se refiere fundamentalmente al dispositivo de percepción dirigida a precisar la dinámica de los objetos, fenómenos y eventos de la realidad.
El investigador observa los hechos, eventos o situaciones con base a su propia experiencia, sensaciones, manera de actuar, y registra en su cognición lo observado, y desde su posición describe la dinámica caracte-rística del mismo (Muñoz y otros, 2011).

\section{Competencias}

analíticas:

el investigador no solo capta, cuestiona $\mathrm{y}$ registra la información, también deberá emprender los procesos de abstracción, análisis e interpretación, para ello, debe hacer uso de un conjunto de técnicas en el análisis, pues sirven de base para desagrupar, decodificar en partes la totalidad del evento; extrayendo cada idea bien sea principal o secundarias.

Competencias metodológicas: consiste en saber aplicar técnicas, tácticas, procedimientos y métodos adecuados a las tareas de investigación, en la que se utilizan atributos de conocimiento, habilidades y destrezas para la elaboración e implementación de diferentes técnicas e instrumentos de recolección de información para la resolución de problemas (Muñoz y otros, 2011).

Competencia Interpretativa: permite al investigador comprender el sentido de un texto cuya acción se encuentra centrada en identificar y reconocer situaciones, problemas o argumentos para el desarrollo y sustento de la acción a investigar (Pérez, y otros, 2012, p. 18).

Competencia de escritura: Apunta hacia la conceptuación de la escritura como un proceso complejo, cuya consustancialidad con la investigación amerita un aprendizaje sistemático, basado en el desarrollo de competencias para el procesamiento de información, la competencia para escribir y hacia la redacción de documentos académicos como espacio intersubjetivo en la relación tutorial. 
En concreto, las competencias investígativas descritas constituyen el eje fundamental de toda indagación, sin embargo, su uso se reduce en la mayoría de los casos a un medio para la búsqueda de información así como la entrega de tareas, trabajos e informes sin la debida atención a la esencia que le compete para precisar los eventos de una realidad (Bucheli, 2010, p. 6).

En este sentido, la formación de competencias investigativas en los estudiantes desde edades tempranas, es una necesidad que posibilita la concreción de habilidades de autoaprendizaje, ya que un estudiante está bien orientado cuando conoce el por qué debe realizar una actividad determinada, qué es lo qué debe hacer y cómo puede controlar por sí mismo la eficiencia de sus ejecuciones (Ferrer, y otros; 2014).

Al mismo tiempo, se resalta que para formar competencias investigativas en los estudiantes, el profesorado debe reorientar la cosmovisión de su proceso de enseñanza aprendizaje, sus representaciones sobre la investigación deben estar orientadas a dejar de lado, la transmisión mecanicista de contenidos, y dar apertura a la idea de hacer ciencia para que se empleen herramientas de análisis, interpretación y conclusiones sobre los eventos que estudian dentro de su contexto. Por tal razón, Barreiro (2015), plantea:

Se establece que para la formación de las competencias investigativas del estudiante se requiere necesariamente de la formación investigativa, en la cual se considere a la investigación como el "proceso esencial" en el proceso de enseñanza y aprendizaje, necesario para la consecución de los objetivos educativos y formativos, como aspecto determinante del desarrollo de la cultura general, y de la formación investigativa del estudiante (p. 61).

Sobre este punto particular, la Ley Orgánica de Educación (2009, p. 18), contempla literalmente en uno de sus fines educativos la promoción de actividades educativas en procura de "desarrollar el potencial creativo de cada ser humano para el pleno ejercicio de su personalidad y ciudadanía", conceptualización que expresa total claridad al tomar en cuenta cada una de las cualidades de la persona desde el punto de vista integral como aspectos que contribuyen al logro de la autorrealización.

Los resultados indican que los docentes de las instituciones estudiadas tienen una actitud positiva hacia la enseñanza de las competencias en investigación, lo cual hace que pueda movilizar desde su experiencia situaciones donde se sienta seguro con respecto a lo que enseña, a creer y confiar en lo que hace y a actuar con seguridad en el proceso educativo para tender un saber investigativo que abra el camino en la cual el estudiante plantee situaciones problemáticas y resuelva las mismas con propiedad.

En definitiva, los resultados encontrados en las tres sinergias cognitiva, afectiva o emocional y conductual dan cuenta a nivel general de una tendencia positiva del docente hacia la enseñanza de competencias investigativas, en la que se propicia el trabajo educativo centrado en el desarrollo de aspectos como observacional, intelectivo, metodológico, analítico, escritural y comunicacional que favorecen el trabajo científico que realizan los estudiantes pertenecientes a las instituciones seleccionadas para el estudio.

Por esta razón, la actitud hacia la enseñanza de competencias investigativas se considera como la disposición general de aceptación y rechazo basadas en la estructura 
cognitiva-emocional y conductual que tiene el docente referido al trabajo científico, donde incluye aspectos intelectivos, observacionales, metodológicos, analíticos, escriturales y comunicacionales.

Al respecto Cotacalla (2010) expresa que el componente cognitivo es el más importante, pues se asegura que sin conocimiento no existe actitud, ya que representa las opiniones y creencias dentro de ciertos límites de certeza.

Igualmente, los resultados del componente afectivo, referido a lo que al docente le gusta enseñar de las competencias en investigación reflejan que la dimensión afectiva es una respuesta esencial en la actitud del docente hacia la investigación porque remite al campo no solo del componente del conocimiento sino del sentimiento, de las preferencias, de las intenciones, de los juicios favorables o desfavorables.

Desde estos resultados, se puede estimar la disposición de los docentes para enseñar al estudiante en el aprendizaje de aspectos referidos a facilitación de métodos para abordar problemas, aspectos lingüísticos y gramaticales, capacidad para preguntar $\mathrm{y}$ formular cuestiones entre otros elementos. Cotacalla, 2010) afirma que:

Hay un lazo entre los componentes cognitivos, particularmente las creencias que manifiestan una cualidad deseable o indeseable, aceptable o inaceptable, y éstas generan la disposición a responder al objeto, específicamente guían nuestro comportamiento. Lo que se infiere que este componente es reconocido como el componente activo de la investigación (p.41).
En ese contexto de la dimensión conductual, se acerca más a un estado en la que el docente se identifica con la labor que debe configurar en su enseñanza sobre competencias en investigación, en la que dispone para ello, de todas las estrategias a su alcance para responder con base a su cognición y emoción el accionar didáctico para favorecer la formación del estudiante.

Estos resultados inducen a afirmar que en las instituciones estudiadas se desarrollan aspectos favorables de la investigación para canalizar el proceso de enseñanza, lo cual permite el logro de competencias en los estudiantes para argumentar, sintetizar y tomar decisiones a favor de la solución de los problemas de la realidad y del contexto.

\section{CONCLUSIONES}

Los resultados de esta investigación conllevaron a concluir que en las instituciones educativas objeto de estudio existe una actitud positiva hacia la enseñanza de las competencias investigativas.

Igualmente, se aprecia con respecto al componente cognitivo $\mathrm{u}$ opinión que los docentes manifiestan una actitud positiva hacia la enseñanza de la investigación, ante lo cual se puede afirmar que los docentes de estas instituciones canalizan sus procesos educativos con actividades que incentiven la investigación.

Con respecto al componente afectivo docentes manifiestan una actitud de aceptación positiva esto indica que los docentes incentivan a los estudiantes a desarrollar sensaciones de afecto por las actividades relacionadas a las competencias investígativas.

Asimismo, con relación a la sinergia conductual los docentes consultados poseen una actitud positiva, por lo que se puede resumir que es un indicador favorable en la enseñanza de competencias investigativas, y 
que está ligada a la disposición de enseñar mediante el uso de estrategias educativas que induzcan a la investigación.

\section{REFERENCIAS}

Acosta, F. (2013).Las actitudes investigativas en la formación escolar. Revista Praxis y Saber. 4 (8). Colombia: Universidad Pedagógica y Tecnológica de Colombia.

Álvarez, I. (2005). Como hacer una tesis en bachillerato. Caracas: Arcaica

Barreiro, P. (2015). Las competencias investigativas del estudiante en el proceso de enseñanza aprendizaje. Revista electrónica de formación y calidad educativa. 182 (2). Ecuador: Universidad laica Eloy Alfaro de Manabí. Manabí: Ecuador.

Bucheli, M. (2010). Desarrollo de competencias investigativas en estudiantes de doctorado a partir del uso de tecnologías de la información y comunicación. 27. Revista CINADE. México: Universidad de Guanajuato.

Cotacalla, D. (2010). Estilos de aprendizaje y actitudes hacia la investigación en los estudiantes de enfermería. Trabajo de Posgrado en Educación de la Universidad Nacional Hermilio Valdizan de Huánuco Universidad Peruana Unión- Perú. UNHV. 2010.

Ferrer, Y., y Ferrer, E. (2014). Estrategias para la formación de competencias investigativas en estudiantes de la carrera de ingeniería informática. Revista Didascalia. 5 (4). Argentina: Universidad de Córdoba.

Finol, M., y Camacho, H. (2008). Competencias investigativas del personal directivo para la ejecución del proyecto educativo integral comunitario (PEIC). Revista multiciencias. 8 (1). Venezuela: Universidad del Zulia.

Hernández, R., Fernández, C. y Baptista, P.
(2010).Metodología de la investigación. Quinta edición. Ciudad de México: México. Mc Graw Hill.

Hurtado de Barrera, J. (2012). El proyecto de investigación. Caracas: Quirón.

Hurtado de Barrera, J. (2012). Metodología de la investigación. Guía para la compresión holística de la ciencia. Caracas: Quirón.

Ley Orgánica de Educación. Gaceta Oficial No. 5.929. 15Dias Del Mes de agosto de 2009. Caracas- Venezuela.

Palacios, G; y Torres, S. (2013). La competencia investigativa en los posgrados de educación: el caso de la maestría en educación, campo formación docente". $1^{o}$ congreso de internacional de investigación educativa. México: Universidad Pedagógica Nacional de Nuevo León.

Pérez, B., Kleeder, J., y Villamizar, U. (2012). Ontología para el desarrollo de la investigación como cultura. Revista enclavos del pensamiento. 12. México: Instituto Tecnológico de estudios superiores de Monterey.

Pérez, M. (2012), Fortalecimiento de las competencias investigativas en el contexto de la educación superior en Colombia. Revista de investigaciones de la UNAD.1.Colombia: Universidad Nacional abierta y a distancia.

Reyes, 0. (2013). Desarrollo de habilidades investigativas de los estudiantes que cursan el bachillerato en línea. Revista mexicana de bachillerato a distancia. 10 (5). México: Universidad nacional Autónoma de México.

Tobón, S. (2008).Gestión curricular y ciclos propedéuticos por competencias. Bogotá: EOOC.

Tobón, S. (2006). Formación basada en competencias. Bogotá: Ecoe 